\title{
BMJ Open Epidurals in Pancreatic Resection Outcomes (E-PRO) study: protocol for a randomised controlled trial
}

\author{
Linda Ma Pak, ${ }^{1}$ Simon Haroutounian, ${ }^{2}$ William G Hawkins, ${ }^{1}$ Lori Worley, \\ Monika Kurtz, ${ }^{2}$ Karen Frey, ${ }^{2}$ Menelaos Karanikolas, ${ }^{2}$ Robert A Swarm, ${ }^{2}$ \\ Michael M Bottros ${ }^{2}$
}

To cite: Pak LM,

Haroutounian S, Hawkins WG, et al. Epidurals in Pancreatic Resection Outcomes (E-PRO) study: protocol for a randomised controlled trial. BMJ Open 2018:8:e018787. doi:10.1136/ bmjopen-2017-018787

- Prepublication history and additional material for this paper are available online. To view these files, please visit the journal online (http://dx.doi. org/10.1136/bmjopen-2017018787).

Received 21 July 2017 Revised 9 November 2017 Accepted 22 November 2017

Check for updates

${ }^{1}$ Department of Surgery, Washington University in Saint Louis School of Medicine, St. Louis, Missouri, USA

${ }^{2}$ Department of Anesthesiology, Division of Pain Management, Washington University School of Medicine, St. Louis, Missouri, USA

Correspondence to Dr Michael M Bottros; bottrosm@wustl.edu

\section{ABSTRACT}

Introduction Epidural analgesia provides an important synergistic method of pain control. In addition to reducing perioperative opioid consumption, the deliverance of analgesia into the epidural space, effectively creating a sympathetic blockade, has a multitude of additional potential benefits, from decreasing the incidence of postoperative delirium to reducing the development of persistent postsurgical pain (PPSP). Prior studies have also identified a correlation between the use of epidural analgesia and improved oncological outcomes and survival. The aim of this study is to evaluate the effect of epidural analgesia in pancreatic operations on immediate postoperative outcomes, the development of PPSP and oncological outcomes in a prospective, single-blind, randomised controlled trial.

Methods The Epidurals in Pancreatic Resection Outcomes (E-PRO) study is a prospective, singlecentre, randomised controlled trial. 150 patients undergoing either pancreaticoduodenectomy or distal pancreatectomy will be randomised to receive an epidural bupivacaine infusion following anaesthetic induction followed by continued epidural bupivacaine infusion postoperatively in addition to the institutional standardised pain regimen of hydromorphone patient-controlled analgesia (PCA), acetaminophen and ketorolac (intervention group) or no epidural infusion and only the standardised postoperative pain regimen (control group). The primary outcome was the postoperative opioid consumption, measured in morphine or morphine-equivalents. Secondary outcomes include patient-reported postoperative pain numerical rating scores, trend and relative ratios of serum inflammatory markers (interleukin (IL)-1 $\beta$, IL-6, tumour necrosis factor- $\alpha$, IL-10), occurrence of postoperative delirium, development of PPSP as determined by quantitative sensory testing, and disease-free and overall survival.

Ethics and dissemination The E-PRO trial has been approved by the institutional review board. Recruitment began in May 2016 and will continue until the end of May 2018. Dissemination plans include presentations at scientific conferences and scientific publications.

Trial registration number NCT02681796.

\section{Strengths and limitations of this study}

- Strengths of this study include its design as a prospective randomised controlled trial and the length of longitudinal follow-up provided postoperatively.

- Limitations include the single-institutional nature of this study.

\section{INTRODUCTION}

Background and rationale

Epidural analgesia

The utilisation of regional analgesia as a compliment to traditional pain management techniques has become an increasingly common practice at many institutions. Placed preoperatively, epidural analgesia provides an important synergistic method of pain control postoperatively. In addition to its usefulness as a pain management adjunct, the deliverance of analgesia into the epidural space, effectively creating a sympathetic blockade, has a multitude of potential additional benefits.

Previous studies have examined the use of epidurals in abdominal surgeries with a small number of retrospective trials focusing on the use of epidurals in pancreatic resections. ${ }^{1}$ While these retrospective studies demonstrated an improvement in patient-reported pain scores postoperatively, objective measures are still needed to quantify these improvements in pain control. ${ }^{2}$ Prior studies have also highlighted a correlation between poor postoperative pain and the development of persistent postsurgical pain (PPSP) ${ }^{3-5}$ As epidural analgesia creates a sympathetic blockade, its intraoperative and postoperative use can mitigate the body's inflammatory response and reduce the activation of peripheral and central nervous system pathways involved in the development of persistent pain syndromes. ${ }^{6}$ Interleukin-1-beta (IL-1 $\beta$ ), IL-6 and tumour necrosis factor-alpha (TNF- $\alpha)$ 
are three pro-inflammatory cytokines involved in the transition from acute pain states to chronic pain syndromes. ${ }^{7}$ IL-10 is an anti-inflammatory cytokine that helps modulate the body's stress response. IL-1 $\beta$, IL- 6 , TNF- $\alpha$ and IL-10, and the relative balance of the pro-inflammatory and anti-inflammatory response, have all been implicated in nociceptive pathways and elevated levels have been found in chronic pain processes. ${ }^{8}$ While our current understanding of the complex modulation pathways of pain is limited, circulating IL-6 has been demonstrated in the upregulation of central and peripheral nociceptive receptors, thereby generating the perception of pain, and potentially establishing the link between acute and chronic pain. ${ }^{910}$ This is of particular importance in our study population of patients with pancreatic diseases for whom adequate pain control is a critical factor in maintaining good quality of life. ${ }^{1112}$

In the immediate postoperative period, the use of epidural analgesia can improve other measures of patient recovery and healing, such as promoting gut motility and reducing the incidence of postoperative delirium. Along with reducing total opioid use, epidural analgesia produces a sympathectomy, allowing for dominance of the parasympathetic system, and further expediting the return of bowel function. ${ }^{13-15}$ With delayed gastric emptying as one of the most common complications and reasons for readmission after pancreatic resections, this valuable benefit of epidural analgesia requires further investigation. ${ }^{16}{ }^{17}$ Delirium is another common postoperative complication that is associated with poor patient outcomes, including functional decline and death, and an effective prophylactic treatment remains to be identified. Through the effects of decreased intraoperative anaesthetic requirement and postoperative opioid use, epidural analgesia may have a potential protective role against postoperative delirium.

The effect of epidural analgesia in suppressing the inflammatory cascade is of particular interest to the field of oncology. In certain types of cancers, including pancreatic, the oncogenic process generates an inflammatory environment that propagates the growth of malignant lesions and continued inflammatory conditions have been implicated in metastatic disease. ${ }^{18-20}$ Pain can further exacerbate systemic inflammation. ${ }^{21}$ In additional to mitigating postsurgical pain, the sympathectomy resulting from epidural analgesia also reduces the body's overall inflammatory conditions. ${ }^{22} 23$ This attenuation of the heightened postoperative inflammatory state of the body may provide an additional means of reducing progression of disease.

\section{Pancreatic diseases}

With improved detection and imaging modalities, the incidence of pancreatic disease, and subsequently, pancreatic operations, has increased. ${ }^{24-26}$ Pancreatic resection continues to be the primary surgical treatment in the treatment of many benign and malignant pancreatic diseases, with an estimated 4000 operations performed annually in the USA. ${ }^{27}$ However, the mean 5-year survival for malignant pancreatic disease remains the lowest of all cancers at $6 \%$, with $70 \%-85 \%$ of patients dying of systemic recurrence, not just local disease. ${ }^{28-30}$ While the search continues for earlier screening methods, the development of adjunctive therapies to surgical resection remains the most promising target of efforts to improve outcomes in malignant diseases of the pancreas. In particular, in recent years, a paradigm shift has occurred in the study of pancreatic malignancies where pancreatic cancer is viewed as a systemic disease, even in early stages, requiring a systemic approach in addition to regional disease control. ${ }^{31-34}$ In previous studies, primarily in prostate and colorectal malignancies, the use of epidural analgesia has suggested a correlation with improved oncological outcomes and survival. ${ }^{35} 36$ Given the role between inflammation and cancer development and recurrence, and the sympathetic blockade created by epidural analgesia, the significance of epidural analgesia in improving oncological outcomes warrants continued investigation.

The aim of this study is to evaluate the effect of epidural analgesia in pancreatic operations on immediate postoperative outcomes, the development of PPSP and oncological outcomes in a prospective, single-blind, randomised controlled trial.

\section{METHODS AND ANALYSIS \\ Study design}

The Epidurals in Pancreatic Resection Outcomes (E-PRO) study is a prospective, single-centre, randomised controlled trial. In total, 150 patients undergoing either pancreaticoduodenectomy or distal pancreatectomy will be randomised to receive an epidural infusion of $0.125 \%$ bupivacaine starting at $5 \mathrm{~mL} /$ hour (range of $5-8 \mathrm{~mL} /$ hour) following anaesthetic induction followed by a standard epidural infusion of $0.1 \%$ bupivacaine at 4-6 mL/hour postoperatively in addition to the institutional standardised pain regimen of hydromorphone patient-controlled analgesia (PCA), intravenous acetaminophen and ketorolac (intervention group) or no epidural infusion and only the standardised postoperative pain regimen (control group). Follow-up information will be collected from the medical record for up to 2 years postoperatively. The study design is outlined in figure 1 .

\section{Eligibility criteria}

Patients 18 years old or older who are able to understand and sign an institutional review board (IRB)-approved informed consent form and who are undergoing either pancreaticoduodenectomy or distal pancreatectomy will be eligible for study inclusion. Patients will be excluded if they fulfil any one of the following criteria: indication for operative intervention being chronic pancreatitis, currently on warfarin with an international normalised ratio (INR) $>1.4$ or clopidogrel that cannot be discontinued 7 days prior 
to surgery, most recent INR prior to surgery $>1.4$, most recent platelet count prior to surgery $<70000 / \mu \mathrm{L}$, chronic opioid use as defined by use of $>20 \mathrm{mg}$ oxycodone, or equivalent, daily, history of pre-existing neuropathic pain conditions, known medical history of significant psychiatric or cognitive impairment, or history of HIV, hepatitis B and/or hepatitis C. Patients will be consented and enrolled during a clinic or preoperative evaluation appointment.

\section{Baseline assessment}

Each study participant will be randomised into the control group with standard of care pain management regiment or the intervention group with the addition of epidural analgesia. Randomisation will occur via a randomised number generation by the principal investigator (PI).

Patients will have the standard of care preoperative evaluation at the Barnes Jewish Hospital Center for Preoperative Assessment and Planning. Routine laboratory tests including complete blood count, comprehensive metabolic panel and coagulation studies will be obtained and reviewed.

In patients receiving chronic antiplatelet or anticoagulant medications, the following procedure will be

\section{Patients $\geq 18$ years of age undergoing pancreaticoduodenectomy or distal pancreatectomy}

Consent

Baseline Assessment

- preoperative anesthesia assessment

- quantitative sensory testing (QST)

- 3D-CAM delirium assessment

- serum inflammatory markers (IL-1b, IL-6, TNF-a, IL-10)

Randomization to Intervention

Intraoperative and postoperative epidural infusion with standard of care pain management regimen (intervention)

\section{Standard of care pain management regimen only (control)}

Postoperative morphine/morphineequivalent consumption (q24 hours)

Measures of postoperative recovery:

- VAS

- intravenous fluid requirements

- anti-emetic doses

- return of bowel function

3D-CAM assessment (POD2)

Serum inflammatory markers (3 hours after surgical incision, POD2, postoperative clinic visit in 2-6 weeks)

QST (postoperative clinic visit in 2-6 weeks)

Figure 1 Study design. IL, interleukin; QST, quantitative sensory testing; TNF, tumour necrosis factor; VAS, visual analogue scores. 
practised to minimise the risk of bleeding (per American Society of Regional Anesthesia and Pain Medicine guidelines ${ }^{37}$ ).

Acetyl salicylic acid (aspirin) or other non-steroidal anti-inflammatory drugs may be continued prior to epidural catheter insertion. Clopidogrel use must be discontinued 7 days before the procedure. The study participant's treating physician (eg, surgeon, cardiologist, neurologist) will be consulted prior to the discontinuation of clopidogrel. Participants receiving warfarin will proceed with the following schedule: if INR $<1.4$, subject may proceed with epidural catheter insertion. If INR $>1.4$, the participant's treating physician will be consulted whether warfarin can be discontinued until INR reaches $<1.4$ or the subject can be switched to low-molecular weight heparin, which can be discontinued 36 hours before catheter insertion. INR and prothrombin time (PTT) will be assessed on the day of epidural catheter insertion in all patients on anticoagulant (but not antiplatelet) therapy.

Study participants will undergo a complete medical history and physical examination, and the following baseline assessments:

1. Evaluation of hypersensitivity or dynamic mechanical allodynia to brush stimulation in the upper abdomen. ${ }^{38}$

2. Quantitative sensory testing (QST) to assess warm and cold detection thresholds, heat and cold pain thresholds, mechanical detection and pain thresholds, presence of wind-up (enhanced temporal summation) to pinprick (online supplementary file 1).

3. Screening for psychological risk factors for acute and chronic pain using Hospital Anxiety and Depression Scale. ${ }^{39}$

4. Baseline assessment for delirium using the 3D-CAM instrument. $^{40}$

5. Baseline assessment of serum inflammatory markers (IL-1 $\beta$, IL-6, TNF- $\alpha$, IL-10).

\section{Interventions}

Postoperatively, all patients will receive a standardised pain regimen including a hydromorphone PCA (initial settings of no bolus dose, $0.25 \mathrm{mg}$ per demand dose, minimal interval dose time of $10 \mathrm{~min}$ ), acetaminophen (1000 mg every 6 hours for 24 hours) and ketorolac ( $15 \mathrm{mg}$ every 6 hours for 72 hours) per surgeon's preference. Study group patients will have an epidural bupivacaine infusion beginning in the operating room (OR).

An epidural infusion of $0.125 \%$ bupivacaine starting at $5 \mathrm{~mL} /$ hour (range of $5-8 \mathrm{~mL} /$ hour) will be started after induction of anaesthesia. Epidural narcotic consisting of fentanyl $50 \mu \mathrm{g}$ will be administered with sterile precaution by the anaesthesia provider before starting the epidural infusion. Epidural boluses of $0.125 \%$ bupivacaine may be administered as guided clinically. A phenylephrine infusion can be used to maintain adequate blood pressure maintaining mean arterial pressures (MAP) $>60 \mathrm{~mm} \mathrm{Hg}$. The epidural infusion can be paused if vasopressor requirements exceed $1 \mu \mathrm{g} / \mathrm{kg} / \mathrm{min}$ of phenylephrine or $0.1 \mu \mathrm{g} / \mathrm{kg} / \mathrm{min}$ of norepinephrine. The epidural infusion is to be paused if haemodynamics become unstable, either due to excessive blood loss or MAP consistently below $60 \mathrm{~mm} \mathrm{Hg}$. The epidural infusion can be resumed when haemodynamics are stable.

The bupivacaine $0.125 \%$ epidural infusion is to be discontinued in the OR at the end of surgery and a standard epidural infusion of $0.1 \%$ bupivacaine at $4-6 \mathrm{~mL} /$ hour will be started in the post-anaesthesia care unit (PACU). The epidural infusion is followed up by an Acute Pain Service in the postoperative period that will titrate the infusion based on the patients' self-reported pain scores and MAP values.

\section{Outcomes}

\section{Primary outcomes}

The primary study outcome is the postoperative consumption of opioids (measured in morphine or morphine-equivalents) in patients undergoing pancreatic resections in the control group compared with the study group. Each subject's morphine or morphine-equivalent consumption postoperatively will be assessed every 24 hours. All subjects will be assessed daily during their postoperative inpatient admission by a trained member of the Acute Pain Service who is blinded to the treatment arm of the study.

\section{Secondary outcomes}

Secondary outcomes of the study include measures evaluated during the inpatient postoperative period as well as during subsequent outpatient follow-up. Study team members blinded to the treatment group of the patient will assess all secondary outcomes. Patient recovery and healing postoperatively will be evaluated using various measures, such as visual analogue scores, intravenous fluid requirements, anti-emetic doses and return of bowel function. Serum inflammatory markers will be evaluated serially, preoperatively on day of surgery, 3 hours after the start of surgical incision in the OR, on postoperative day 2 (POD2) and at the initial postoperative visit 2-6 weeks after surgery. Postoperative delirium assessments will be performed when patients can be aroused sufficiently in order to be assessed for delirium (Richmond Agitation-Sedation Scale > -4). Each patient will be assessed for delirium on POD2 as postoperative delirium typically first manifests 24-96 hours after surgery. For non-verbal patients, the Confusion Assessment Method for the Intensive Care Unit (CAM-ICU) instrument will be used, and for verbal patients, the 3D-CAM instrument will be used. ${ }^{40}$ As delirium is a fluctuating disorder and can be missed with sporadic delirium assessments, a structured method of chart review will be used to complement the clinical assessments.

This combined approach (3D-CAM interview or CAM-ICU plus chart review) increases the sensitivity and retains specificity in detecting incident delirium. The trial staff has undergone formal training in 
clinical delirium assessment and on the chart review methodology.

Patients will be seen for their initial postoperative weeks at 2-6 weeks after hospital discharge and will undergo repeat PPSP evaluation at that time.

Patients will continue to be followed in clinic for 2 years postoperatively with laboratory and radiological evaluation as deemed appropriate by the primary surgeon. Patients will be followed for tumour recurrence and overall survival. Data will be collected directly from subject's medical record; no study-specific procedures will be implemented at follow-up visits.

\section{Sample size}

Sample size estimation was performed based on the study primary outcome of postoperative opioid consumption. Based on our prior experience, this estimation will be based on the following assumptions ${ }^{41}$ : expected morphine consumption is $30 \mathrm{mg}$ intraoperatively, $30 \mathrm{mg}$ on postoperative day 1 (POD1), $20 \mathrm{mg}$ on POD2 and $10 \mathrm{mg}$ on POD3. Therefore, expected total morphine consumption in the first 72 hours is, on average, $80 \mathrm{mg}$. Then, assuming that the SD of morphine consumption is $30 \mathrm{mg}$, that a $20 \mathrm{mg}$ difference in morphine consumption between groups is a clinically meaningful reduction of opioid use and assuming normal distribution of morphine consumption in both patient groups, the proposed sample size for $a=0.05$ and $b=0.2$ would be 37 patients per group ( 74 patients in total). However, we propose to increase the sample size of the study to 150 total patients to account for patients lost to follow-up, inability to complete the scheduled pancreatic resection, data errors and other unanticipated study problems.

\section{Recruitment}

Participants will be recruited primarily through the Washington University Hepatobiliary-Pancreatic Surgery clinics. Subjects will be given verbal (initially) and then written descriptions of the study aims, procedures, risks and benefits, and will be required to give written informed consent. A member of the investigative team provides all study descriptions, informed consent and answers all questions. No deception is required for the purposes of this study. All subjects will be aware of the randomisation used in this study to either the control or intervention group. Subjects are informed verbally and in writing that participation is voluntary and they may refuse to participate and may withdraw from the study at any time without penalty.

\section{Allocation}

Participants will be randomised in a 1:1 ratio into the control group with standard of care pain management regimen or the intervention group with the addition of epidural analgesia. Randomisation will occur via randomised number generation.

This is a single-blind study. Patients and the primary investigative team will be aware of the randomisation.
However, all study members performing data collection will be blinded to the randomisation.

\section{Data analysis and management}

Data analysis for this study will focus on the comparison of patient outcomes (postoperative morphine/ morphine-equivalent consumption, measures of postoperative recovery, inflammatory markers, 3D-CAM/ CAM-ICU assessments, QST) between the intervention and control study groups. Based on data distribution, continuous variables will be compared between the two groups using Student's t-test or the Mann-Whitney U test as appropriate. When appropriate, significance of findings will be adjusted for multiple comparisons using the Bonferroni correction method.

The Center for Biomedical Informatics at Washington University will be used as the central location for data collection and management. Since 2008, Washington University has hosted Research Electronic Data Capture (REDCap), a secure, web-based application for building and storing online research and clinical trial databases. The REDCap servers are securely housed in an on-site limited access data centre managed by the Center for Biomedical Informatics at Washington University. All web-based information transmission is encrypted, and all data are stored on a private firewall-protected network. All users are assigned individual user IDs, and passwords and individual access is restricted on a role-specific basis. REDCap was developed specifically around Health Insurance Portability and Accountability Act (HIPAA) guidelines and is also implemented and maintained in accordance with institutional security guidelines.

\section{Monitoring}

The study team will monitor all study participants for adverse events. The PI will report all unanticipated problems or adverse events, all conditions of non-compliance and any new information that may affect the continued or current enrolment of study participants to the IRB. All events will be reported to the IRB within 10 working days of the event or of notification of the PI of the event. The death of a study participant must be reported to the IRB within one working day of the event or of notification of the PI of the event.

The specific monitoring plan for this investigation is commensurate with the risks and the size and complexity of the investigations planned. The potential risks are attributable to performing insertion of the epidural catheter and the use of bupivacaine for neuraxial analgesia. Based on these considerations, the monitoring plan involves engaging a colleague from the Department of Anesthesiology not involved in the study to serve in a monitoring capacity. Based on the small size and relatively low-risk nature of the protocol, only a third person (the colleague), rather than a full Data Safety Monitoring Board, will be used. The colleague will be an anaesthesiologist knowledgeable in the risks associated with nerve blocks and local anaesthetic administration. This 
individual will review the annual summary of adverse events. In addition, this colleague will review all reports of a serious adverse event or an unexpected adverse event.

\section{ETHICS AND DISSEMINATION}

\section{Ethics approval and consent}

The E-PRO trial was provided ethical approval by the Washington University in St. Louis's IRB which serves Washington University and Barnes-Jewish Hospital. Study recruitment and enrolment began in May 2016 and will continue through the end of 2017. Potential study participants will be given verbal and then written descriptions of the study aims, procedures, risks and benefits, and written informed consent will be obtained for all participants. All participants are informed verbally and in writing that participation is voluntary and they may refuse to participate and may withdraw from the study at any time without penalty.

\section{Confidentiality}

Only the investigators and research team will have access to any protected health information of study participants and any study data. All subjects will be assigned a study ID number. All study data and samples will be coded with the assigned study ID number. A key to the code linking code numbers to patient names will be kept at a separate location, under lock and key; this link will be destroyed at the conclusion of this study. All data will be recorded by a member of the research team and will be stored in a password-protected electronic database stored on the departmental network drive. Study data will not be entered into participants' medical records.

\section{Dissemination}

Dissemination plans include presentations at scientific conferences and scientific publications.

\section{CONCLUSIONS}

This trial investigates a wide spectrum of potential benefits to patients undergoing pancreatic resection. During the initial postoperative period, the use of epidural analgesia can aid in improving postoperative pain control, decreasing opioid consumption, reducing the incidence of delirium and expediting recovery. In addition to improving immediate postsurgical pain control, epidural analgesia may reduce the development of PPSP, which can persist for weeks to years after surgery. Lastly, epidural analgesia can help reduce the body's stress response to a major operation, which has been linked to malignant progression and spread.

Based on this trial, we seek to establish the role of epidural analgesia as part of the standard of care in future patients undergoing pancreatic operations.

Contributors LMP and MMB are the primary authors of the E-PRO protocol. Their contributions include conceptualising the study design, drafting and editing the protocol, and creating the electronic database REDCap used for data collection.
WGH contributed to the E-PRO protocol by editing the protocol and recruiting patients for enrolment. SH contributed to the E-PRO protocol by conceptualising the study design, drafting and editing the protocol, and supervising data collection. LW contributed to the E-PRO protocol by editing the protocol, recruiting patients and collecting data. MKu contributed to the E-PRO protocol by recruiting patients and collecting data. KF contributed to the E-PRO protocol and editing the protocol, creating the electronic database REDCap, coordinating patient enrolment and data collection. MKa contributed to the E-PRO protocol by conceptualising the study design and editing the protocol. RAS contributed to the E-PRO protocol by conceptualising the study design and editing the protocol. All authors have critically revised the E-PRO protocol and approved the final version. All authors agree to be accountable for the accuracy and integrity of all aspects of the E-PRO trial.

Funding QThis works was supported by the Foundation for Barnes-Jewish Hospital Project Award grant number 8083-88.

Competing interests None declared.

Patient consent Obtained.

Ethics approval This study has been approved by the institutional review board at Washington University in St. Louis.

Provenance and peer review Not commissioned; externally peer reviewed.

Open Access This is an Open Access article distributed in accordance with the Creative Commons Attribution Non Commercial (CC BY-NC 4.0) license, which permits others to distribute, remix, adapt, build upon this work non-commercially, and license their derivative works on different terms, provided the original work is properly cited and the use is non-commercial. See: http://creativecommons.org/ licenses/by-nc/4.0/

C Article author(s) (or their employer(s) unless otherwise stated in the text of the article) 2018. All rights reserved. No commercial use is permitted unless otherwise expressly granted.

\section{REFERENCES}

1. Park WY, Thompson JS, Lee KK. Effect of epidural anesthesia and analgesia on perioperative outcome: a randomized, controlled Veterans Affairs cooperative study. Ann Surg 2001;234:560-71.

2. Choi DX, Schoeniger LO. For patients undergoing pancreatoduodenectomy, epidural anesthesia and analgesia improves pain but increases rates of intensive care unit admissions and alterations in analgesics. Pancreas 2010;39:492-7.

3. Kehlet $\mathrm{H}$, Jensen TS, Woolf CJ. Persistent postsurgical pain: risk factors and prevention. Lancet 2006;367:1618-25.

4. Katz J, Seltzer Z. Transition from acute to chronic postsurgical pain: risk factors and protective factors. Expert Rev Neurother 2009;9:723-44.

5. Pogatzki-Zahn EM, Schnabel A, Zahn PK. Room for improvement: unmet needs in postoperative pain management. Expert Rev Neurother 2012;12:587-600.

6. Zhang JM, An J. Cytokines, inflammation, and pain. Int Anesthesiol Clin 2007;45:27-37.

7. DeVon HA, Piano MR, Rosenfeld AG, et al. The association of pain with protein inflammatory biomarkers: a review of the literature. Nurs Res 2014;63:61-2.

8. Uçeyler N, Eberle T, Rolke R, et al. Differential expression patterns of cytokines in complex regional pain syndrome. Pain 2007;132:195-205.

9. De Jongh RF, Vissers KC, Meert TF, et al. The role of interleukin-6 in nociception and pain. Anesth Analg 2003;96:1096-103.

10. Woolf CJ, Costigan M. Transcriptional and posttranslational plasticity and the generation of inflammatory pain. Proc Natl Acad Sci U S A 1999;96:7723-30.

11. Burger V, DOlimpio JT. Improving quality of life through pain control. Clin J Oncol Nurs 2013;17:117-8.

12. Chan C, Franssen B, Domínguez I, et al. Impact on quality of life after pancreatoduodenectomy: a prospective study comparing preoperative and postoperative scores. J Gastrointest Surg 2012;16:1341-6.

13. Carroll J, Alavi K. Pathogenesis and management of postoperative ileus. Clin Colon Rectal Surg 2009;22:047-50.

14. Leslie JB, Viscusi ER, Pergolizzi JV, et al. Anesthetic routines: the anesthesiologist's role in Gl recovery and postoperative ileus. Adv Prev Med 2011. doi: 10.4061/2011/976904. [Epub ahead of print 29 Dec 2010]

15. Person B, Wexner SD. The management of postoperative ileus. Curr Probl Surg 2006;43:6-65. 
16. Emick DM, Riall TS, Cameron JL, et al. Hospital readmission after pancreaticoduodenectomy. J Gastrointest Surg 2006;10:1243-53.

17. Ahmad SA, Edwards MJ, Sutton JM, et al. Factors influencing readmission after pancreaticoduodenectomy: a multi-institutional study of 1302 patients. Ann Surg 2012;256:529-37.

18. Mantovani A, Allavena P, Sica A, et al. Cancer-related inflammation. Nature 2008;454:436-44.

19. Marchesi F, Monti $P$, Leone BE, et al. Increased survival, proliferation, and migration in metastatic human pancreatic tumor cells expressing functional CXCR4. Cancer Res 2004;64:8420-7.

20. McKay CJ, Glen P, McMillan DC. Chronic inflammation and pancreatic cancer. Best Pract Res Clin Gastroenterol 2008;22:65-73.

21. Laird BJ, Scott AC, Colvin LA, et al. Cancer pain and its relationship to systemic inflammation: an exploratory study. Pain 2011;152:460-3.

22. Bar-Yosef S, Melamed R, Page GG, et al. Attenuation of the tumor-promoting effect of surgery by spinal blockade in rats. Anesthesiology 2001;94:1066-73.

23. Liu S, Carpenter RL, Neal JM. Epidural anesthesia and analgesia. Their role in postoperative outcome. Anesthesiology 1995;82:1474-506.

24. Balcom JH, Rattner DW, Warshaw AL, et al. Ten-year experience with 733 pancreatic resections: changing indications, older patients, and decreasing length of hospitalization. Arch Surg 2001;136:391-8

25. Winter JM, Cameron JL, Campbell KA, et al. 1423 pancreaticoduodenectomies for pancreatic cancer: a singleinstitution experience. J Gastrointest Surg 2006;10:1199-211.

26. Matrisian L, Aizenberg R, Rosenzweig A. The alarming rise of pancreatic cancer deaths in the United States. http://www.pancan. org/section_research/reports/incidence_report.php (accessed 13 Mar 2014)

27. Cress RD, Yin D, Clarke L, et al. Survival among patients with adenocarcinoma of the pancreas: a population-based study (United States). Cancer Causes Control 2006;17:403-9.

28. Siegel R, Ma J, Zou Z, et al. Cancer statistics, 2014. CA Cancer J Clin 2014;64:9-29.

29. Jemal A, Siegel R, Ward E, et al. Cancer statistics, 2006. CA Cancer J Clin 2006;56:106-30.
30. Hishinuma S, Ogata Y, Tomikawa M, et al. Patterns of recurrence after curative resection of pancreatic cancer, based on autopsy findings. J Gastrointest Surg 2006;10:511-8.

31. Gnerlich JL, Luka SR, Deshpande AD, et al. Microscopic margins and patterns of treatment failure in resected pancreatic adenocarcinoma. Arch Surg 2012;147:753-60.

32. Sohal DP, Walsh RM, Ramanathan RK, et al. Pancreatic adenocarcinoma: treating a systemic disease with systemic therapy. J Natl Cancer Inst 2014;106:dju011.

33. Scavonetto F, Yeoh TY, Umbreit EC, et al. Association between neuraxial analgesia, cancer progression, and mortality after radical prostatectomy: a large, retrospective matched cohort study. $\mathrm{Br} \mathrm{J}$ Anaesth 2014;113(Suppl 1):i95-102.

34. Holler JP, Ahlbrandt J, Burkhardt E, et al. Peridural analgesia may affect long-term survival in patients with colorectal cancer after surgery (PACO-RAS-Study): an analysis of a cancer registry. Ann Surg 2013;258:989-93.

35. Christopherson R, James KE, Tableman M, et al. Long-term survival after colon cancer surgery: a variation associated with choice of anesthesia. Anesth Analg 2008;107:325-32.

36. Cummings KC, Xu F, Cummings LC, et al. A comparison of epidural analgesia and traditional pain management effects on survival and cancer recurrence after colectomy: a population-based study. Anesthesiology 2012;116:797-806.

37. Horlocker TT, Wedel DJ, Rowlingson JC, et al. Regional anesthesia in the patient receiving antithrombotic or thrombolytic therapy: American Society of Regional Anesthesia and Pain Medicine Evidence-Based Guidelines (Third Edition). Reg Anesth Pain Med 2010;35:64-101.

38. Finnerup NB, Sørensen L, Biering-Sørensen F, et al. Segmental hypersensitivity and spinothalamic function in spinal cord injury pain. Exp Neurol 2007;207:139-49.

39. Zigmond AS, Snaith RP. The hospital anxiety and depression scale. Acta Psychiatr Scand 1983;67:361-70.

40. Ely EW. Vanderbilt University. Confusion assessment method for the ICU (CAM-ICU): the complete training manual. 2014. http://www. icudelirium.org/docs/CAM_ICU_training.pdf (accessed 14 Dec 2016).

41. Mann C, Pouzeratte Y, Boccara G, et al. Comparison of intravenous or epidural patient-controlled analgesia in the elderly after major abdominal surgery. Anesthesiology 2000;92:433. 